\title{
SHELL FORMATION IN NGC 474
}

\author{
A.J. TURNBULL \\ University of Hertfordshire, Hatfield, Herts, UK \\ D. CARTER \\ Liverpool John Moores, Byrom Street, Liverpool, UK \\ T.J. BRIDGES \\ Royal Greenwich Observatory, Cambridge, UK \\ AND \\ R. C. THOMSON \\ University of Hertfordshire, Hatfield, Herts, UK
}

We present broad band photometry (B and R) of the classic shell galaxy NGC 474. Preliminary results indicate that the shells have a similar colour to and follow the same trend of colour with radius as the underlying galaxy.

\section{Introduction}

NGC 474 is a classic shell galaxy originally catalogued as peculiar by Arp (1966), appearing in his atlas as Arp 227, together with its nearby spiral companion NGC 470. Both galaxies have the same redshift, as measured in $\mathrm{HI}$ of 2372 and $2374 \mathrm{~km} \mathrm{~s}^{-1}$ respectively (de Vancouleurs et al. 1991) and, assuming both lie at a distance of $31 \mathrm{Mpc}$, have an apparent separation of some $45 \mathrm{kpc}$. The shells around NGC 474 are unusually bright, making this a prime target for detailed photometric studies.

Several models have been put forward to explain the origin of shells, of which the most successful are the merger model (Quinn 1984; Dupraz \& Combes 1986; Hernquist \& Quinn 1988, 1989) and the interaction model (Thomson \& Wright 1990; Thomson 1991). According to the merger model, shells are formed by the phase wrapping of stars accreted from the disrupted companion galaxy. In this case, the shell colours may be different to that of the underlying host galaxy. According to the interaction model, shells are density waves induced in a thick disk population during an interaction (not a merger) with another galaxy. Consequently, the shell colours are expected to be the same as that of the underlying host galaxy. 


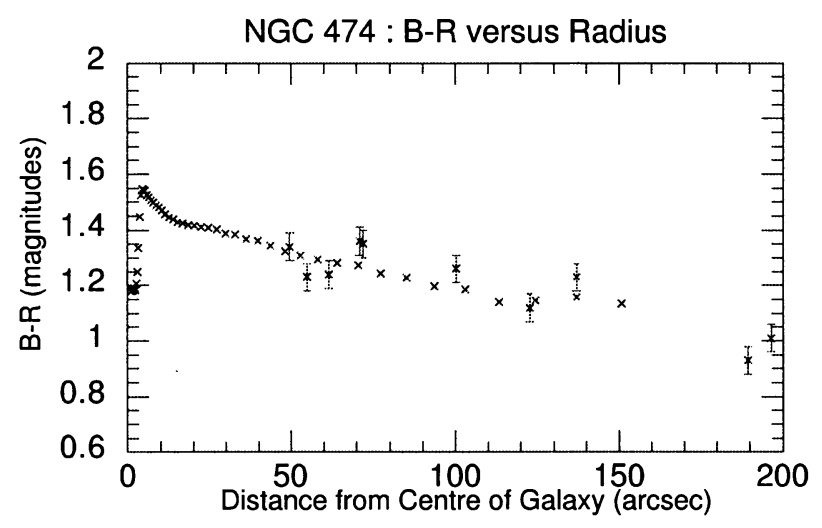

Figure 1. Derived B-R Colours for NGC 474 and Shells.

\section{Observations and Data Reduction}

The images of NGC 474 were obtained at prime focus on the WHT(B) and INT(R) on La Palma. Standard IRAF data reduction methods were used. The underlying galaxy profiles were obtained using the ISOPHOTE package in IRAF. Using a fixed size aperture $\left(6^{\prime \prime} \times 6^{\prime \prime}\right)$ and residual sky subtraction the shell magnitudes in $B$ and $R$ were determined from the galaxy subtracted image. The derived galaxy colours (crosses) and shell colours (crosses with estimated errors) are plotted in Fig. 1.

\section{Discussion}

There appears to be a significant colour gradient with the host galaxy becoming bluer at larger radii. Each shell's B-R colour is similar to that of the underlying galaxy at the same radius. This is naturally explained by the interaction model whereas the merger model requires the special condition that the merged galaxy is of the same colour as NGC 474. A parallel N-Body study is underway.

\section{References}

Arp, H. 1966, ApJS, 14, 1

de Vancouleurs et al. 1991, Third Reference Catalog of Bright Galaxies (RC3)

Dupraz, C., \& Combes, F. 1986, $A \& A$, 166, 53

Hernquist, L., \& Quinn, P. 1988, ApJ, 331, 682

Hernquist, L. \& Quinn, P. 1989, ApJ, 342, 1

Thomson, R.C. 1991, MNRAS, 253, 256

Thomson, R.C. \& Wright, A.E., 1990, MNRAS, 247, 122

Quinn, P. 1984, $A p J, \mathbf{2 7 9}, 596$ 